Editorial

\title{
Fundación SERMES para la Investigación Clínica
}

\section{SERMES Foundation for Clinical Research}

\author{
Josefina Berlanga \\ Directora de Fundación Sermes. Madrid. España. \\ Recibido: 28-08-14 \\ Aceptado: 02-09-14
}

\section{Correspondencia}

Josefina Berlanga

Directora de Fundación Sermes

Rufino González 14, Esc. 1. ' 2. D

28037 Madrid. España.

Tel: + 3491375.69 .30

Fax: + 3491375.69 .31

Correo electrónico: jberlanga@fundacionsermes.org

www.fundacionsermes.org

Resumen

SERMES se constituyó en 1997 como un Centro Especial de Empleo, con una plantilla inicial de siete trabajadores con experiencia en grabación y proceso de datos. Su objetivo principal siempre ha sido la integración laboral de personas con discapacidad. En 2001 cuando se fundó SERMES CRO ${ }^{\mathrm{TM}}$ para apoyar el desarrollo y crecimiento de la empresa en todas las actividades relacionadas con Investigación Clínica.

Con el objetivo de ampliar la cartera de clientes internacionales y dar servicio clientes locales en proyectos internacionales, SERMES CRO funda PSN con oficinas centrales en EEUU y subsidiarias en España, Francia, Inglaterra, Alemania, Italia, Holanda y Dinamarca además de tener acuerdos de colaboración con otras compañías para cubrir aquellos países en los que no se posee de oficinas propias. PSN es una CRO global que ofrece a empresas farmacéuticas, biotecnológicas e instituciones independientes una alternativa flexible, rápida, de calidad y coste-efectiva.

Por otro lado, se ha creado SERMES UK, en Inglaterra, con el fin de ofrecer la representación legal a promotores sin filial ni ubicación física en la Unión Europea (UE) y aprovechar las sinergias propias de una localización en Inglaterra. Dicha representación, conocida como "representación legal», es válida para los ensayos clínicos con medicamentos, así como los ensayos clínicos de dispositivos médicos.

Para más información sobre Fundación Sermes para la Investigación Clínica, por favor contactar:

Tel 91.375.69.30 - Fax 91.375.69.31

info@fundacionsermes.org

www.fundacionsermes.org

www.sermescro.com

www.psnglobal.org

Med Segur Trab (Internet) 2014; 60 (236) 475-479

Palabras clave: Fundación, Cátedra, Premio al Puesto Mejor Adaptado en la Empresa, Discapacidad, Formación. 


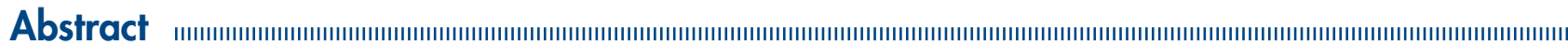

The Foundation SERMES for the Clinical Investigation was based on June of 2012 in response to the anxieties of the Director of Sermes Planning to try to alleviate, in the measure of our possibilities, the deficiencies and inequalities with the ones that the people with disability in the labor world are found. We believe firmly, that the formation is a vehicle of independence and development, and a fundamental and inalienable right. Qualifying the people in abilities that permit them to develop a full professional life is something that collaborates in the development of the individual but also in that of the company. Our Mission is to promote the presence of disabled personnel in the labor market through integral formation. As Vision we pursue the integration of disabled people in the labor world through the formation, as well as the promotion of the clinical investigation in those matters that contribute value and innovation to the company. Because of it our Values are centered in the disabled one, in the flexibility in the actions, in promoting the culture of the wok, in the labor integration since the equality and the affirmative action offering a continuous monitoring and attention personalized, in the investigation and social formation, in the innovation constant in formation and matters and in the commitment with the Excellence.

Med Segur Trab (Internet) 2014; 60 (236) $475-479$

Keywords: Foundation Chair, Award for Best Adapted Since the Company, Disability, Education. 
La Fundación Sermes para la Investigación Clínica se fundó en junio de 2012 como respuesta a las inquietudes del Director de Sermes Planificación para intentar paliar, en la medida de nuestras posibilidades, las deficiencias y desigualdades con las que se encuentran las personas con discapacidad en el mundo laboral. Creemos firmemente, que la formación es un vehículo de independencia y desarrollo, y un derecho fundamental e inalienable. Capacitar a las personas en habilidades que les permitan desarrollar una vida profesional plena es algo que colabora en el desarrollo del individuo pero también en el de la sociedad.

Nuestra Misión es potenciar la presencia de personal discapacitado en el mercado laboral a través de formación integral. Como Visión perseguimos la integración de personas discapacitadas en el mundo laboral a través de la formación, así como la potenciación de la investigación clínica en aquellas materias que aporten valor e innovación a la sociedad. Por ello nuestros Valores se centran en el discapacitado, en la flexibilidad en las actuaciones, en potenciar la cultura del trabajo, en la integración laboral desde la igualdad y la discriminación positiva ofreciendo un seguimiento continuo y atención personalizada, en la investigación y formación ecosocial, en la innovación constante en formación y materias y en el compromiso con la Excelencia.

Teniendo en cuenta todo esto, durante este tiempo hemos realizado las siguientes actividades (incluimos las más relevantes):

- Premio al Puesto Mejor Adaptado en la Empresa. Este año hemos celebrado su segunda edición. Este certamen se ha creado para premiar a aquella empresa que, cumpliendo los requisitos que marca la ley, va más allá asegurando el bienestar de todos los trabajadores y más concretamente de los que tienen un mayor número de hándicaps a la hora de realizar su tarea.

Nuestra pretensión ha sido otorgar un certificado de calidad a aquella empresa que vaya más allá de lo exigido, para que los empleados con alguna discapacidad puedan desempeñar su trabajo con las mejores garantías.

Hemos buscado la Excelencia en el compromiso con las personas discapacitadas y premiar el esfuerzo realizado a tres bandas: la empresa, los compañeros y la persona o personas discapacitadas.

Creemos que es importante visibilizar el esfuerzo que realizan tanto las empresas como los trabajadores para integrar y normalizar una situación excepcional. No son importantes las capacidades que no tenemos sino las que si poseemos y nos hacen desarrollar una vida profesional y personal plena.

Hemos contado, en las dos ediciones, con la colaboración de FREMAP y Discert en el comité asesor que valora las candidaturas finalistas. Y con Fundación «la Caixa» en la cesión del espacio (CaixaForum) para la entrega de los premios.

Entregamos un premio y dos accésit que en el 2013 recayeron por este orden en GREEMOIL, S.L. como premiado y Accenture, Magnolia T.V. como accésit; y en el 2014 el premio recayó en TASUBINSA y los accésit en Alentis Servicios Integrales y Megino, S.L.

En ambas ediciones tanto el número como la calidad de los proyectos presentados han sido importantes, resultando difícil la elección.
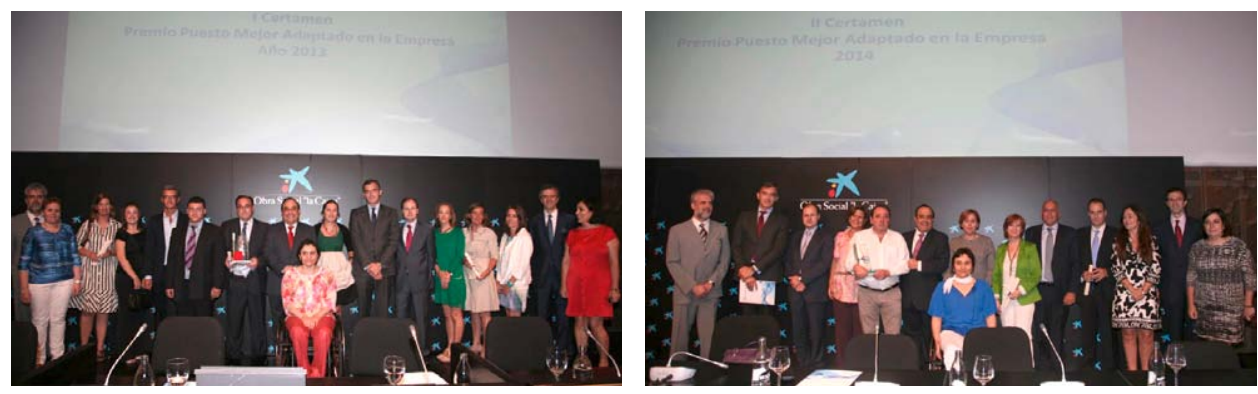
- En el 2013 hemos desarrollado dos acciones formativas subvencionadas por el Ministerio de Trabajo a través del Servicio Público de Empleo Estatal que pretendían fomentar la empleabilidad de jóvenes desempleados (personas con discapacidad, desempleados con baja cualificación, desempleados de larga duración...), dando formación a 256 alumnos de todo el territorio nacional.

- Hemos impartido Cursos de Especialista en Gestión Administrativa en Investigación Clínica (curso presencial de $94 \mathrm{~h}$. con prácticas no laborales en la empresa), uno de ellos becado plenamente a personas con discapacidad, derivadas de la Consejería de Familia y Asuntos Sociales a través de los Centros Base de la Comunidad de Madrid.

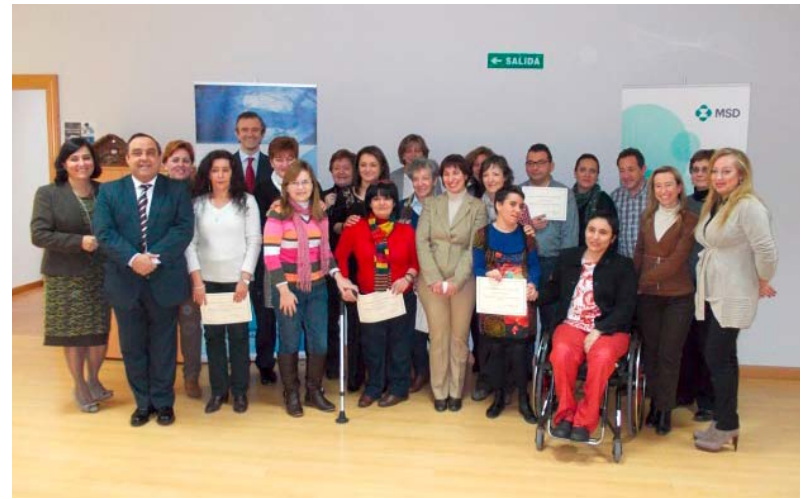

- Hemos creado la Cátedra UAM-Fundación Sermes para el estudio de la discapacidad de causa Neurológica, a través de la cual aportamos fondos para una investigación sobre lesionados medulares que se está llevando a cabo en el Hospital Puerta de Hierro por el Dr. Vaquero, director de la Cátedra.

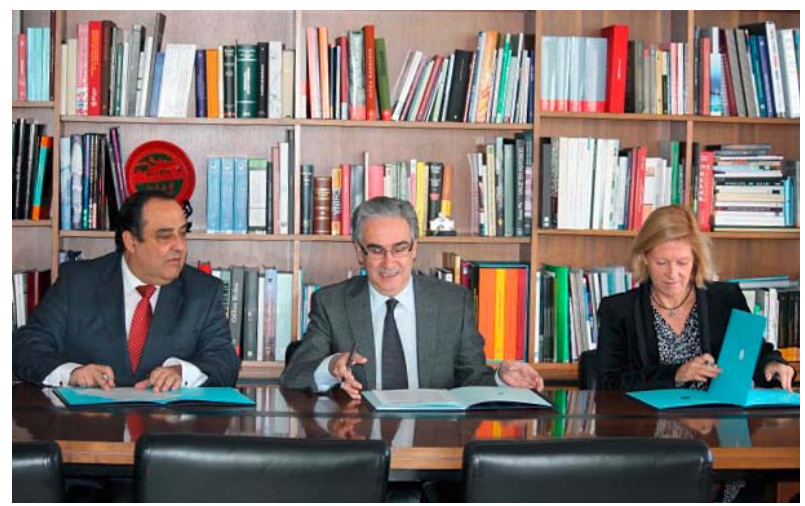

- Hemos firmado dos convenios de colaboración con la UAM para colaborar en la formación e inserción laboral de alumnos con discapacidad de dicha Universidad, centrándonos principalmente en el área biosanitaria.

- Otra de las actividades que se han desarrollado a lo largo de estos años ha sido el patrocinio de InvestigArte. Es un concurso de arte visual científico y tecnológico dirigido a dos perfiles específicos:

- Grupos de investigación

- Alumnos de ESO, Bachillerato y Formación profesional

Ha sido ideado por un grupo de investigadores apasionados de la fotografía y su utilidad para explicar a las nuevas generaciones y a la sociedad en general, las innovaciones científicas y tecnológicas asociadas a sus proyectos científicos. 
El concurso está organizado por EducaBarrié (el canal de comunicación con la comunidad educativa de la Fundación Barrié), Campus Vida (el Campus de Excelencia Internacional Liderado por la Universidad de Santiago de Compostela), y el Instituto de Investigaciones Sanitarias de Santiago (IDIS); todos ellos con el objetivo principal de acercar la ciencia y la tecnología a nuestra sociedad, a través de un concurso que pretende activar la visión artística y creativa de la ciencia de alumnos de ESO, Bachillerato y Formación Profesional así como de los propios investigadores.

- Hemos participado en el Día Internacional de las Personas con Discapacidad y el Día Internacional de las Personas con Esclerosis Múltiple con relatos de personas que han compartido con nosotros su experiencia y que han sido publicados en nuestra web.

- Para no perder de vista el momento por el que estamos pasando hemos lanzado campañas solidarias de recogida de juguetes y alimentos y su posterior reparto entre personas de especial vulnerabilidad.

- Actualmente estamos inmersos en el lanzamiento de una Jornada sobre Terapias Avanzadas que va a tener lugar el día 8 de octubre en la Escuela Nacional de Sanidad donde contamos con grandes expertos en el tema desde el área de la regulatoria, pasando por investigadores, empresas y pacientes, tanto del ámbito público como privado.

Nada de esto hubiera sido posible sin el apoyo de la empresa matriz que lanzó idea de la creación de la Fundación a través de su Director General. 